\title{
5. Discussion
}

This study has presented trends in the prescribing of stimulant medication for the treatment of ADHD in children and adolescents in NSW. Where comparisons are available, the patterns are generally consistent with overseas patterns of prescribing.

Over the last decade there has been a significant increase in the prescribing of stimulant medication for the treatment of ADHD in children in NSW. This finding is consistent with what is known to have occurred elsewhere. For example, analysing data on the number of office-based visits to physicians in the US, Robison et al. (1999) found a 2.9-fold increase in the rate of patients aged 5-18 years diagnosed with ADHD who were prescribed stimulant medication from 1990 to $1995 .{ }^{57}$ Similarly, Zito et al. (2000) reported a three-fold increase in stimulant treatment for preschoolers during the early 1990s in regions of the US. ${ }^{58}$ In The Netherlands, Schirm et al. (2001) reported a five-fold increase in the use of stimulant medication among 0-19 years from 1995 to 1999.59

The sharpest increases in NSW occurred in the mid-1990s. There are many factors that may have contributed to this, including: an increased public and clinician awareness and acceptance of ADHD as a disorder; a broadening of the diagnostic criteria for ADHD; a greater knowledge of the course of the disorder; an increase in the availability of ADHDspecific services, particularly paediatric and child psychiatric services; an increase in knowledge about the use and effectiveness of stimulant medication; fewer interruptions in treatment because of reduced concerns about growth retardation; lengthier periods of treatment; and an increase in the use of stimulant medication for the treatment of adults. In NSW specifically, this period saw a greater emphasis on professional collaboration between clinicians and educators. The publication Talk, Time, Teamwork, which facilitates and supports the work of teachers and doctors in the management of ADHD, was developed and distributed to schools across NSW in $1995 .^{73}$

Results of this study suggest that current rates of prescribing of stimulant medication in NSW are not cause for alarm. At the end of 2000, approximately one per cent of children were being treated with stimulant medication for ADHD. This is somewhat lower than the US, where is it estimated that three per cent of children are treated with stimulants. ${ }^{56,60}$ Moreover, if it is accepted that the prevalence of ADHD in Australia is around 11 per cent, ${ }^{26}$ it can be argued that children in NSW are being undertreated; ${ }^{9}$ only one in every 10 children with ADHD is undergoing what is known to be the single most effective form of treatment for ADHD.

In NSW, the prevalence of stimulant treatment is greatest among school-age children, particularly in the ages eight to 13 years. This is not surprising given that it is usually soon after a child commences formal schooling that the presence of ADHD becomes apparent. Indeed, results from this study indicate that the number of children aged five to eight years commenced on stimulant medication for the first time has increased over the last decade relative to other children.

The rate of treatment of very young children, or preschoolers, appears to be low in NSW. As at 1 December 2000, fewer than one in 1,490 children aged less than five years was on stimulant medication in NSW. Over the last decade, the growth in the number of preschoolers treated with stimulant medication has been less than the average growth in the number of children overall on stimulant medication.

${ }^{\mathrm{q}}$ As indicated earlier, the prevalence rate is likely to be over inclusive. The National Survey of Mental Health and Wellbeing, from which it was obtained, did not strictly apply DSM-IV criteria; children with ADHD-type symptoms due to anxiety disorder or abuse, for example, may have been included in the prevalance rate. 
Concerns have been expressed about preschoolers being treated with psychotropic medication for a number of reasons: the medications are not approved for use in the very young; there are very few controlled data showing whether they are effective in this age group; psychiatric diagnoses in preschoolers generally lack validity and reliability-clinicians must rely on parental reports and it is difficult to establish whether the behaviours are outside the range of age-appropriate behaviours; there is little knowledge and considerable apprehension about the long-term effect of psychotropics on the developing brain; and there are scarce data about the pharmacokinetic and pharmacodynamic characteristics of these drugs in the very young. ${ }^{74}$

In NSW, a number of requirements are imposed on clinicians to ensure that prescribing of stimulant medication for very young children with ADHD is appropriate. These include requirements to submit written applications supported by clinical reports, to provide written progress reports, and, in cases where the child is aged two years, requirements to obtain a second opinion on the appropriateness of stimulant medication.

The present examination of individual patient approvals granted by the Department in the 18-month period to June 2000 indicates that stimulant medication is effective, at least to some extent, for a significant proportion of two- to three-year olds (Table 4). It should be noted that the data presented here on two- to three-year olds are not necessarily representative of all two- to three-year olds commenced on stimulants. In this study, details of a large number of three-year olds who were commenced on stimulant medication were not available for analysis. This was mainly because the child turned four years of age during the medication trial period and therefore an individual application was not required, or because medication was not continued after a trial period and no information was provided by the clinician regarding the effectiveness of the medication during the trial.

In about 39 per cent of the cases involving two- to three-year olds that were examined in the present study, side effects were reported. The most commonly reported were appetite and emotional problems. About 21 per cent evidenced no side effects, indicating that perhaps as high as 79 per cent of these children may have had side effects. Once again, caution should be used as these figures may not be representative of all two- to three-year olds. However, this rate of side effects is fairly consistent with the published literature, where some side effects have been reported in 80 per cent of children. ${ }^{63}$ Generally the side effects children experience are mild and temporary.

The level of use of non-stimulant medication among two- to three-year olds that was found in this study is of concern. Although non-stimulant medications such as clonidine are reported to be useful in the management of some ADHD-related behaviours, the safety and efficacy of these drugs in very young children are yet to be demonstrated. ${ }^{33,74}$

The use of psychotropic medication in very young children is not uncommon. In regions in the US, Zito et al. (2000) reported a large increase in the use of nonstimulant medication in the early 1990s for the treatment of ADHD in preschoolers. ${ }^{58}$ The increase in the use of clonidine was particularly large. The use of antidepressants also increased.

Rappley et al. (1999) examined claims for outpatient and pharmacy services in Michigan, US, involving children with ADHD aged three years or younger. ${ }^{75}$ They found that 57 per cent of these children had received psychotropic medication. Twenty-two different psychotropic medications had been used with the group. Methylphenidate was the most common medication used, followed by clonidine, and dexamphetamine. Over a third of the children were treated simultaneously with two or three medications. Preliminary findings from a recent survey of Australian paediatricians and child psychiatrists indicate 
that 'off-label' prescribing (that is, prescribing for conditions for which the medication does not have marketing approval) for children in Australia is widespread. ${ }^{76}$

In Australia, medications other than stimulants that may be used to treat ADHD, such as antidepressants and clonidine, are not formally monitored and are not restricted to specialist prescribing. The mechanisms that are in place to monitor the use of stimulants have no jurisdiction over these other medications. ${ }^{11}$ The decision to prescribe the medication, therefore, rests solely with the clinician.

In the present study, the age distribution of children on stimulant medication was found to be similar for boys and girls (Tables 1 and 2). However, in almost all age groups, boys outnumbered girls by about four to one. This gender disparity is a common finding in ADHD research. It is thought that some of the disparity occurs because boys are more likely than girls to be referred for treatment due to their higher rates of disruptive behaviour. ${ }^{29}$ The data presented in this study lend some support to this notion, especially in the case of very young children. Among the two- to three-year olds, males outnumbered females more than seven to one. It is among this age group that oppositional defiant behaviours, for example, are highly prevalent with ADHD and are more commonly displayed by boys than by girls. ${ }^{27}$ It is clearly recognised that children who display aggressive and defiant behaviour at a very early age have generally poor long-term outcomes, therefore it is important to detect and treat these children as early as possible. ${ }^{77}$

Although boys outnumber girls in treatment rates, the growth in the number of girls commenced on stimulant treatment in the last decade has been greater than the growth in the number of boys. The increase from 1990 to 2000 in the number of girls commenced on medication was 1.3 times the average increase in children commenced on medication. An increase in the number and proportion of girls on medication has been observed overseas. ${ }^{55,59}$ As a greater understanding and acceptance of ADHD has occurred over time, a greater number of girls may have been recognised as having the disorder and referred for treatment. The disproportionate growth in girls on psychostimulant therapy is likely to continue in the future if it is accepted that the prevalence rate of ADHD for girls in Australia is about half of that for boys. ${ }^{26}$

This study has reported some interesting findings with respect to the drug used in psychostimulant therapy for ADHD. In the very early 1990s, more children who commenced stimulant treatment in NSW were started on methylphenidate (Figure 5). This most likely reflects US practice where methylphenidate was, and still is, the most common stimulant prescribed. From 1993, however, more NSW children were commenced on dexamphetamine than methylphenidate. The fact that more children are commenced on dexamphetamine is probably, at least in part, due to economic reasons. Over the last decade, dexamphetamine has been available on the Pharmaceutical Benefits Scheme whereas methylphenidate has not been, making dexamphetamine a considerably less expensive product for the family. ${ }^{\mathrm{r}}$

While more children may be commenced on dexamphetamine, roughly the same number of children at any one time are treated with methylphenidate as are treated with dexamphetamine (Figure 8). This may mean that a higher proportion of children commenced on dexamphetamine drop out of treatment compared with those commenced on methylphenidate. A more likely explanation is that a proportion of children commenced on dexamphetamine are switched from dexamphetamine to methylphenidate. It is well documented that some individuals find one stimulant drug to be more effective than another. ${ }^{35,50}$

Regional differences in the rate of psychostimulant therapy have been observed elsewhere, and this was the case for NSW. The rate of children on stimulant medication 
at the end of 2000 varied considerably according to the health area involved. The region with the highest rate (Hunter Health area) had a rate almost 15 times the region with the lowest rate (Far West Health area). Differences between the other health areas were less marked, with rates ranging from 4.5 per 1,000 people aged 2-17 years in the Central Sydney area, to 15.1 per 1,000 people aged 2-17 years in the Central Coast area.

Without further investigation it is not possible to conclusively say what factor or factors contributed to these differences. However, a number of factors were likely to be involved. One of these factors concerns the availability of treatment services. For example, in the Far West area, a region characterised by geographical remoteness, access to ADHDspecific services is very limited. Other factors that may have contributed to differences in regional treatment rates include the prevalence of ADHD symptoms and variations in practice patterns concerning diagnostic criteria and methods; the clinician's approach to the treatment of ADHD, and more particularly his or her philosophy toward the use of psychostimulant therapy; socioeconomic factors; and parental and familial attitudes toward health services and treatment strategies.

The regional differences found in this study for older children provide some support for the involvement of socioeconomic factors. For children in the age group 16-17 years, the regional distribution of rates of treatment was quite dissimilar to that seen for the other age groups (Figure 17 versus Figures 14-16). For 16-17 year olds, Northern Sydney area and South East Sydney area had the highest rates of treatment. These areas are characterised by relatively high income, low unemployment, and high levels of education. ${ }^{78}$ One could speculate that significant impairment among older adolescents in these regions is related to high expectations of academic achievement and potential. This may be particularly relevant if the inattentive type of ADHD, associated with higher level language and processing difficulties, is disproportionately prevalent among these older adolescents.

It has been shown in this study that psychostimulant therapy is a long-term strategy for managing ADHD for a significant number of children with the disorder. As at 1 December 2000, about one in four children with ADHD undergoing psychostimulant therapy in NSW had been receiving this treatment for more than three years. This is not surprising given that ADHD is a chronic disorder.

However, for an equally significant proportion of children with ADHD, psychostimulant therapy is not an option that is pursued beyond a short period. Data presented here indicate that about 20 per cent of children who start stimulant treatment discontinue treatment after their first prescription (Table 5). This is consistent with the fact that stimulants are not an effective treatment for at least 10 per cent of children with ADHD.

For older children, short-term attrition from stimulant treatment appeared to be somewhat greater than that for younger children (Figure 18). Almost 40 per cent of 14 and 15 year olds dropped out of treatment after their first prescription. There are a number of possible explanations for this.

It is possible that the older children found stimulant medication less effective than children of other ages. Other family influences such as quality of family functioning and family socioeconomic adversity may have also played a role. Factors more likely to have been involved include the child's dislike and fear of taking medication, the child's age at initiation of treatment, and the presence of comorbid conditions, especially oppositional defiant disorder and conduct disorder.

${ }^{r}$ The cost of methylphenidate can be as much as four times the cost of dexamphetamine. 
Generally, the treatment of adolescents is regarded as more difficult. They may choose not to continue treatment because taking the medication gives them a sense of inadequacy, or because they feel somehow that their parents are trying to control their behaviour. ${ }^{79}$ They may judge the medication to be of little benefit, despite the views of others. For example, while parents may perceive the effects of medication to be favourable, children taking the medication do not always agree. ${ }^{80}$ Older children are more likely to discontinue medication than younger children because, at this age, they are more autonomous and less influenced by their parents, who would usually decide on treatment options. Children with comorbid oppositional defiant disorder are also more likely to discontinue medication than other children with ADHD; children who are defiant are less likely to comply with requests to take medication. ${ }^{81}$

While the focus of this study has been on the use of stimulant medication, it is important to recognise that stimulants are only one option available for the management of ADHD. There is little doubt from published research that stimulants are very effective for many children at improving many of the symptoms of ADHD. Psychostimulant therapy, however, is also effective only while it is being used. Individual children will have different responses, with very few exhibiting gains across-the-board. Many children receiving stimulants may still retain more behaviour problems than normal children, and some children will not be helped by medication at all.

The consensus among experts is that stimulant medication should be used in conjunction with non-medication interventions. By temporarily controlling symptoms, stimulants help people to pay more attention and help them to better organise themselves. In this way, they facilitate the use of other kinds of strategies or treatments that can improve academic performance, social integration and family harmony. Compared with medication treatment alone, a combined treatment approach can lead to a greater number of children being successfully treated ${ }^{54}$ and lower dosages of medication being required. ${ }^{35}$ The combined approach may also be more effective for treating children with multiple comorbid disorders. ${ }^{82}$

In Australia, the National Health and Medical Research Council (NHMRC) (1997) has recommended that treatment for ADHD should be 'multimodal and involve consideration of simultaneous medication use, behaviour management, family counselling and support, educational management, and specific development issues' relevant to the child involved (page 38). ${ }^{11}$ They also recommend that 'specific and individualised treatment plans should be formulated for each child with ADHD and their family' (ibid.). These and other recommendations of the NHMRC are incorporated into criteria used by clinicians in NSW for the diagnosis and management of ADHD in children and adolescents. ${ }^{72}$

The decision to treat a child with ADHD with stimulant medication largely rests with the child's parent(s) on the advice of the treating clinician. They must weigh up the likely benefits, such as alleviation of core symptoms, and probable reduced risk of later academic and social problems, against the potential hazards, including likely, but probably minor, side effects and indeterminate long-term effects.

To facilitate this decision, clinicians must ensure that comprehensive assessment and diagnostic procedures are used. They must fully explain to parents, as well as children where appropriate, the nature of stimulant treatment. The importance of regularly reviewing the child and the management strategies in place must be recognised. If strategies are to be used to their full potential, they must be adjusted as needed, and steps taken to ensure that parents and children are complying with instructions.

A major challenge for health authorities is ensuring that statutory obligations are met with respect to the prescribing of stimulant medication, without impeding the 
delivery of an appropriate and effective form of treatment for ADHD. This study has demonstrated that the regulatory and monitoring system in place in NSW supports and encourages stimulant prescribing that is in accord with modern, appropriate and effective management of ADHD. 